\title{
Fault Classification System for Computer Networks using Fuzzy Probabilistic Neural Network Classifier (FPNNC)
}

\author{
Karwan Qader, Mo Adda \\ University of Portsmouth, School of Computing, \\ Buckingham Building, Lion Terrace, PO1 3HE Portsmouth, Great Britain \\ \{karwan.qader,mo.adda\}@port.ac.uk
}

\begin{abstract}
Over the last decade, the world has witnessed the rapid development of networking applications of different kinds, and network domains have become more and more advanced regarding with their level of heterogeneity, complexity and the size. Some obstacles such as availability, flexibility and insufficient scalability have affected the existing centralized network management systems, as networks become more distributed. In this work a Fuzzy Probabilistic Neural Network Classifier (FPNNC) is proposed, comprising a hybrid fault classification algorithm based on Fuzzy Cluster Mean (FCM) with Probabilistic Neural Network (PNN) to classify the detected fault datasets. These results will assist network administrators with a highly effective tool to classify faults that occur in computer network systems, enabling them to take well-informed decisions pertaining to security, faults and performance.
\end{abstract}

Keywords: Clustering, classification, network faults, fault diagnosis, FCM, PNN, FPNNC

\section{Introduction}

With the rapid development of computer network technology, the scale and function of networks is constantly increasing. The increasing importance and complexity of networks led to the development of network fault management as a distinct field, providing support for network administrators with quality services and ensuring that networks work appropriately. Fault diagnosis is a central aspect of network fault management. Since faults are unavoidable in communication systems, their quick detection and isolation is essential for the robustness, reliability and accessibility of the system. In large and complex communication networks, automating fault diagnosis is critical. Because of many factors, including the volume of network information, it is hard to solve network fault problems with traditional tools, rendering intelligent diagnosis a critical method in the process of network fault diagnosis [1].

In the process of network fault diagnosis, both cluster and classifier techniques play a significant role by identifying types and locations of the faults. The use of clustering in grouping objects is one of the most commonly used data mining techniques. The resultant groups of objects can help a network administrator to take accurate decisions to protect data communications over a network. The method based on back propagation 
(BP) technique is most extensively used in intelligent diagnosis method of artificial neural network [2]. Statistics show that $80 \%$ of neural network models have adopted BP network or its variants. However, the neuron numbers of the BPs imply layer are attained by experience, not from precise computing of theory, and the BP neural network has several shortcomings, such as falling into local least point easily and needing a long time for training [3].

Network PNN is an extensively used artificial neural network. Its structure is simple and its training succinct. The advantage of PNN lies in finishing the work with the linear study algorithm, which was previously, achieved using nonlinear study algorithm.

This article is organized as follows. The following section reviews related studies, followed by explanation of the proposed algorithm Fuzzy Probabilistic Neural Network Classification (FPNNC), with discussion of the network characteristic parameters and patterns. Section 4 describes the case studies and the source of the datasets. The results are presented and discussed in section 5. Finally, the conclusion of the paper is presented in section 6 .

\section{Related Work}

In recent years, much research has been undertaken to explore network faults, particularly fault diagnosis and management. However, a recent review study has shown that although the trends of fault management and diagnosis have been increasingly explored in recent research papers, most of them do not include contributions about the fault diagnosis in computer network system. This paper addresses this gap by extending prior work focused on faults classification to practical application for computer network system.

A number of studies have been carried out and methods have been proposed in the field of fault detection and classification for semiconductor manufacturing equipment [4]. One such method put forward by [5], detects faulty processes of the semiconductor manufacturing equipment using its data, recognizes anomalies and classifies the root cause of the faults by reading the production equipment data, which consists of all such information; albeit this can be quite useful, it is challenging to study due to the complexity and volume of data [6]. Hence, this paper explores Modular Neural Network modelling, whereby data from production equipment is aggregated into associated subsystems, enabling Fault Detection and Classification(FDC) using Dempster-Shafer (DS) method to consider the ambiguities in fault detection. The method employs Radio Frequency (RF) power source module probing, which is advantageous for detecting the chamber leak simulation and helps in classifying the faults by evaluating the RF probe voltage signals. This paper was successful in justifying the use of D-S theory by successful fault detection at subsystem level, with no missed alarms [7] [8].

Fault detection is one of the most important network management tasks, as analysed by [9], to propose a statistical method based on Wiener filter to capture the abnormal changes in the behaviour of the MIB variables. The algorithm of the study took data from two different scenarios and four different case studies. Such an analysis provided 
the manager node of the network high level of information instead of huge data volume [9]. The study in [10] of Sensor Fusion and Sensor Fault Detection with Fuzzy Clustering presented an effective approach for multi-sensor fusion and fault diagnosis, which makes use of FCM algorithms for separating the signals. The fusion engine in turn generates a fused signal based on the concept of centre of gravity (COG) defuzzification method. In this approach, the sensor fault detector is designed from the total fused signal residual and the output of the sensors. The results of the simulation showed a clear improvement in the fault detection accuracy [10]. A two-phase approach for measuring the performance of the cluster based internet services was presented by [11]. The first phase of the methodology employs the fault-injection approach for measuring the impacts of faults on the network performance while the second phase makes use of analytical models to assess the network performance by combining the measurements of first phase and the fault loads [12]. Such a two-phased approach lets the evaluator study how the servers respond to various design-related decisions, rate of faults and other factors. Four versions of PRESS web servers were tested against five fault classes to measure the performance of the servers in different scenarios [11] [13].

The area of smart networks has been the subject of good amount of research and review recently because of the concept of computational intelligence, which is incorporated into smart networks [14]. Because of computational intelligence, smart networks are capable of detecting their faults and classifying them [15]. Keeping in view this capability of smart networks, [16] presented two techniques for fault detection and classification in power transmission lines in smart networks. The techniques proposed are based on Quarter Sphere Support Vector Machine (QSSVM). The first approach makes use of Temporal Attribute QSSVM, utilizing the temporal, and attributes correlations of the measurements of the transmission lines for detection of faults during the stage of transient. The second approach makes use of Attribute QSSVM (A-QSSVM) and takes into account attribute correlations for the automatic fault detections and classifications [17], the results of these two approaches displayed accuracy in fault detection and classification (as high as 99\%), which amounted to a significant reduction in terms of computational complexity compared to traditional techniques making use of multi class SVM for fault detection and classification [18]. Additionally, as compared to the traditional methods, these techniques are still quite unsupervised and can be available for implementation on the existing fault-monitoring infrastructure for limited online supervision in power systems of smart networks [16].

An ensemble Fault Diagnosis Based on Fuzzy C-means Algorithm of the Optimal Number of Clusters and Probabilistic Neural Network (FCM-ONC-PNN) represented by [19] portrayed the significance of fault diagnosis as a process being followed for maintaining quality of the products in industrial systems and ensuring various aspects like reliability, safety and efficiency from the point of view of operations in many plants.

Finally Fuzzy C-Means Clustering and Feed Forward Neural Network was used by [20] to find the fault-proneness of a software module, focusing on the benefits of the early detection of fault prone software components and how hybrid approach based on fuzzy C-means clustering based approach and feed-forward neural network based approach can be used to find faults. The proposed method of the current study rely on FCM and PNN. The main reasons behind selecting these two techniques refers to their 
robust factors. Fuzzy Cluster Means (FCM) is the most suitable algorithm among clustering techniques due to its robust characteristics to deal with network fault diagnosis problems, such as handling unclear boundaries of clusters, overcoming high dimensionality problems and its flexibility, especially for traffic analysis, which is an iterative optimal algorithm [21][22]. Likewise, PNN maintains excellent characteristics such as high precision of the non-linear algorithm. The corresponding weights values of PNN are the distribution of the model sample, and also the network does not need training, therefore it can meet the requirements of real-time processing [2]. Moreover, its robust characteristic to sample noises, fast speed of its training data and the accuracy rate of the classification, enhance achieved results to have a higher quality [23].

\section{Proposed Network Faults Classification Algorithm (FPNNC)}

The Fuzzy Neural Probabilistic Neural Network Classification (FPNNC) is proposed to classify the faults that occur in the computer network system. The developed algorithm comprises the combination of two techniques, Fuzzy Clustering Means (FCM) as unsupervised learning technique and Probabilistic Neural Network (PNN), as shown in fig. 1 .

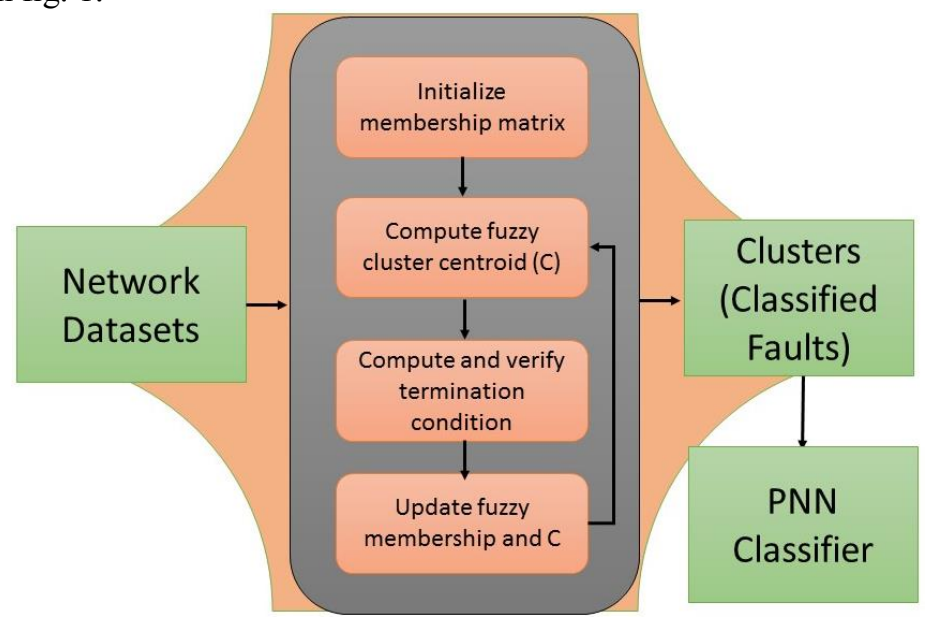

Fig. 1. Schematic overview of the proposed approach (FPNNC)

FCM consists mainly of four phases used to generate clustered feature vectors from the data points of each specific fault; the featured vectors are then forwarded to PNN in order to classify the fault types.

\subsection{FCM}

Fuzzy C-means (FCM) is one of the most common unsupervised clustering methods that was originally proposed by Bezdek in 1981. As with other clustering techniques, 
FCM primarily relies on measuring the distance between data points and it uses Euclidean distance to measure similarity between objects. The distance measure helps the algorithm to make decisions to create groups for each data point depending on the similarity and dissimilarity between points. Similar data points are kept in a group known as a cluster. Similarity is assayed with values between 0.0 and 1.0 ; the value 0.0 indicates highly dissimilar, while 1.0 indicates the highest similarity between objects. As illustrated in figure 1, the system takes the data points as input then generate the clusters based on four main phases.

The Euclidean Distance Function, which is known as objective function $\mathrm{J}$ m is used in FCM to get fuzzy $\mathrm{C}$ partition $A=\left\{A_{1}, A_{2}, A_{3}, \ldots, A_{n}\right\}$ for given dataset $X=$ $\left\{X_{1}, X_{2}, X_{3}, \ldots X_{n}\right\}$ and number of clusters denoted by "c". The main objective in FCM is to minimize $\mathrm{J} \_\mathrm{m}$ depicted in the following equation in order to get the optimal clusters. As it is an iterative method, it has to achieve better minimization in each iteration.

$$
J_{m}(\mu, V: X)=\sum_{i=1}^{c} \sum_{j=1}^{n}\left(\mu_{i j}\right)^{m}\left\|X_{j}-V_{i}\right\|^{2}
$$

Where $\mu_{i j}$ represents the membership function of data point $X_{j}$ in the ith cluster. $\mathrm{m}$ is the fuzzifier which acts as the fuzziness controller value; generally $\mathrm{m}$ is any real number that is greater $1 . V_{i}$ is the centre of ith cluster, whereas $\|$.$\| denotes the$ Euclidean distance.

The equation (1) is used to compute the value of $J_{m}(\mathrm{U}, \mathrm{V})$ and determine the criterion function based on a threshold. Firstly, the membership function and cluster centroids are randomly initialised with some constraints consideration:

$$
\sum_{i=1}^{c} \mu \mu_{i j}=1
$$

In order to optimise the results in each iteration through an iterative process, the fuzzy membership and fuzzy cluster centroids are updated using the equations shown below:

$$
\begin{gathered}
V_{i}=\left(\sum_{j=1}^{n}(\mu i j)^{m} X_{j}\right) /\left(\mu_{i j}\right)^{m} \\
\mu_{i j}=\left[\sum_{k=1}^{c}\left(\left\|X_{j}-V_{i}\right\|^{2} /\left\|X_{j}-V_{k}\right\|^{2}\right)^{1 /(m-1)}\right]^{-1}
\end{gathered}
$$

\subsection{PNN}

Probabilistic Neural Network (PNN), which was introduced by D.F. Specht in the early $1990 \mathrm{~s}$, is one of the common feed-forward neural networks used in classification problems [24]. The general architecture of PNN is organized based on multi-layered 
feed-forward network into four different layers (input layer, hidden layer, pattern layer or summation layer and output layer), as shown in fig. 2.

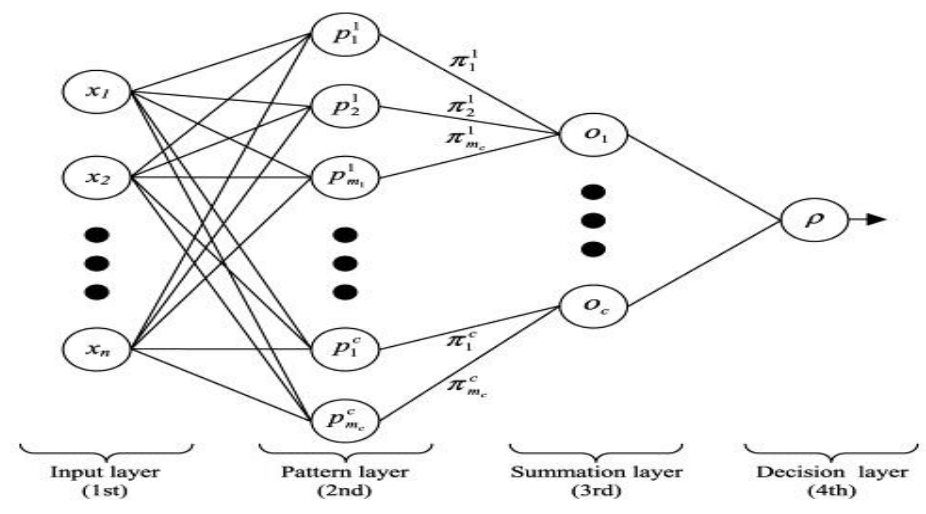

Fig. 2 Diagram of PNN [25]

The hidden and summation layers, known as the radial basis layer, refers to all equations applied to the input neurons, which calculate the distance from input vectors to training input vectors. Competitive (summation) layer is considered to be another main layer that compares the weighted vectors and select the maximum one to predict the target category. The number of neurons in summation layer is equal to the number of train set neuron.

The Euclidean Distance function used to calculate the distance and to indicate how close the input is to a training sets in the pattern layer as shown below:

$$
D_{j}=|| W_{i j}-X|| \cdot b
$$

Where b represent to Radial Basis Function (RBF), which can be used to adjust the susceptibility of the radial basis neurons by having different values and set to:

$$
b=\frac{[-\log (0.5)]^{1 / 2}}{\text { Spread }}
$$

Spread is the extended coefficient of RBF. Moreover, the Radial Basis Function (RBF) as shown in equation (7), is applied to calculate the probability of each inputted neuron $(\mathrm{X})$ by comparing the input vectors with weight neuron vectors $W_{i j}$.

$$
R_{j}=\operatorname{radbas}\left(D_{j}\right)
$$

Out of several candidate functions of RBF, a Gaussian function has been selected for the proposed work.

$$
R_{j}=e^{-D_{j}^{2}}
$$


Thus, the output relies on the distance between $W_{i j}$ and $\mathrm{X}$; it inverts with the distance, thus when the distance decreases it increases to reach its maximum while $W_{i j}=X$.

\section{The Characteristic of Network Faults}

SNMP protocols use Management Information Base (MIB) to obtain knowledge about the status information of computers or devices in network in order to manage objects in the right manner. MIB includes any statistical and status information about each node in the system. It has information about counters at each node interface and uses them to indicate the number of packets or octets that have been sent, dropped, received and delivered. Each device has one or more interface.

The total functions of SNMP rely on MIB, which follows the ISO standards and is defined by RFC 1155, which locates the network devices. In 10 groups of MIB, 10 variables out of $178 \mathrm{MIB}$ variables were selected from interface and IP groups. The variables in these two groups are more likely to have more sensitivity with traffic. Table 1 shows all selected parameters in IF and IP group that enable the system to diagnose faults easily.

Table 1. Characteristic Parameters of Network

\begin{tabular}{cc}
\hline No. & Network Parameter \\
\hline P1 & ifInNUcastPkts22 \\
P2 & ifInOctets22 \\
P3 & ifInUcastPkts22 \\
P4 & ifOutNUcastPkts22 \\
P5 & ifOutOctets22 \\
P6 & ifOutUcastPkts22 \\
P7 & ipForwDatagrams22 \\
P8 & ipInDeliver22 \\
P9 & ipInReceive22 \\
P10 & ipOutRequests22 \\
\hline
\end{tabular}

\section{Case Study and Results Discussion}

The proposed work complements and extends past research work detecting, classifying and collecting network faults. The datasets were captured in two different (heavy and light) scenarios using MIB variables from IP and IF groups for four different types of traffic. The main aims to collect data in two different scenarios is to check the capability of the system in terms of whether it can detect the traffic faults in different environment or not. The two scenarios were created by changing the size of packets and bandwidth. ifInUcastPkts, ifInNUcastPkts, ifOutUcastPkts and ifOutNUcastPkts are more affected 
by traffics than the other selected variables. In addition, the experiment tested the router as the first gate to attack the server as the main target. The router differs from the server in that its variables are limited to network layer group (IP group). In contrast, in the case of a server, one can read most of the MIB variables, starting from a low-level group such as the interface group, to an application layer group.

The current model is applied on four different types of network traffic, which includes server crash or server link failure, broadcast storm, babbling node, and normal traffic. Matlab was used to give different outputs and results based on FCM and PNN for the available datasets. After all data points were inputted to the system, FCM generated clusters of featured vectors for each dataset. The empirical results show all available clusters when there is a failure in the link to server or server crash.

Consequently, PNN creates a model to classify these vectors properly, which they attain by FCM. First, all target classes' indices are converted to vectors, in order to compare them with the input vectors. After consecutive mathematical calculations, the system starts testing the network on the design input vectors. This make the network simulation and converts its vector outputs to indices.

The proposed system was tested and applied on another three different types of network traffic datasets. The results obtained from the preliminary simulation of the proposed work shows different output patterns for each specific faults.

Overall, these results indicate that the proposed algorithm FPNNC is able to classify all inputted fault datasets properly and provide distinct output patterns for each specific fault traffic. Consequently, it gives a clear vision to network administrator to realize about the location and the type of faults.

\section{Conclusion}

The key aspect of network fault management is the process of a fault classification, by which it concludes the details of a failure from a set of tested failure indications. This paper has given an account of and the reasons for the widespread use of FCM and PNN in classification problems. The main purpose of the current study was to use new proposed technique (FPNNC) to classify the faults properly in a computer network system. The experiment results show that FPNNC is able to classify the faults in the real-time system and categorize them into different patterns outputs based on the types of fault that occur. Consequently, these results provide a level of security and performance for the network system, helping network administrators to have a clear vision for the problems based on visualization of classified faults and to take decisions faster related to security, performance and faults.

\section{References}

1. Fenton, W. G., McGinnity, T. M., \& Maguire, L. P.: Fault diagnosis of electronic systems using intelligent techniques: a review. Systems, Man, and Cybernetics, Part C: Applications and Reviews, IEEE Transactions on, 31(3), 269-281 (2001) 
2. Gao, Y., \& Zhou, X.: The design of network fault diagnosis system based on PNN. Paper presented in 2nd International Conference at the Future Computer and Communication (ICFCC), (2010)

3. Zheng, Q., Qian, Y., \& Yao, M.: A network event correlation algorithm based on fault filtration PRICAI 2006: Trends in Artificial Intelligence, pp. 864-869 Springer (2006)

4. Barakat, M., Druaux, F., Lefebvre, D., Khalil, M., \& Mustapha, O.: Self-adaptive growing neural network classifier for faults detection and diagnosis. Neurocomputing, 74(18), 3865$3876(2011)$

5. Hong, S. J., Lim, W. Y., Cheong, T., \& May, G. S.: Fault Detection and Classification in Plasma Etch Equipment for Semiconductor Manufacturing-Diagnostics. Semiconductor Manufacturing, IEEE Transactions on, 25(1), 83-93 (2012)

6. Rengaswamy, R., \& Venkatasubramanian, V.: A fast training neural network and its updation for incipient fault detection and diagnosis. Computers \& Chemical Engineering, 24(2), 431$437(2000)$

7. Chao, C.S., \& Liu, A.C.: An alarm management framework for automated network fault identification. Computer communications, 27(13), 1341-1353 (2004)

8. Hong, S. J., Lim, W. Y., Cheong, T., \& May, G. S.: Fault Detection and Classification in Plasma Etch Equipment for Semiconductor Manufacturing-Diagnostics. Semiconductor Manufacturing, IEEE Transactions on, 25(1), 83-93 (2012)

9. Al-Kasassbeh, M., \& Adda, M.: Network fault detection with Wiener filter-based agent. Journal of Network and Computer Applications, Elsevier 32(4), 824-833 (2009)

10.ElMadbouly, E., Abdalla, A., \& ElBanby, G. M.: Sensor fusion and sensor fault detection with fuzzy clustering. In International Conference presented at the Computer Engineering and Systems (ICCES), 2010

11.Nagaraja, K., Li, X., Bianchini, R., Martin, R. P., \& Nguyen, T. D.: Using Fault Injection and Modeling to Evaluate the Performability of Cluster-Based Services. Paper presented at the USENIX Symposium on Internet Technologies and Systems (2003)

12.Song, Y.-H., Johns, A., Xuan, Q., \& Liu, J.: Genetic algorithm based neural networks applied to fault classification for EHV transmission lines with a UPFC, pp. 278-281 (1997)

13.Zhang, B., He, Z., \& Qian, Q.: Application of wavelet entropy and adaptive nerve-fuzzy inference to fault classification. In International Conference of Power System Technology, PowerCon, pp. 1-6. IEEE, (2006)

14. Youssef, O. A.: An optimised fault classification technique based on Support-VectorMachines. Paper presented at the Power Systems Conference and Exposition, PSCE'09. IEEE/PES, (2009)

15. Bouloutas, A. T., Calo, S., \& Finkel, A.: Alarm correlation and fault identification in communication networks. Communications, IEEE Transactions on, 42(234), 523-533 (1994)

16. Shahid, N., Aleem, S., Naqvi, I. H., \& Zaffar, N.: Support Vector Machine based fault detection \& classification in smart grids. Paper presented at the Globecom Workshops (GC Wkshps), IEEE, (2012)

17.Mahamedi, B.: A novel setting-free method for fault classification and faulty phase selection by using a pilot scheme. In 2nd International Conference of the Electric Power and Energy Conversion Systems (EPECS), (2011)

18. Chutani, S., \& Decotignie, J.D.: A perspective on fault diagnosis of industrial communication networks. In IEEE International Workshop of the Factory Communication Systems, WFCS'95, Proceedings, (1995)

19.Yang, Q., Guo, J., Zhang, D., \& Liu, C.: Fault Diagnosis Based on Fuzzy C-means Algorithm of the Optimal Number of Clusters and Probabilistic Neural Network. International Journal of Intelligent Engineering \& Systems, 4(2), 51-59 (2011)

20.Dashora, K., Kriti, P., Dalal, P., \& Panwar, D. A.: Software Fault Prediction Using Fuzzy CMeans Clustering and Feed Forward Neural Network. International Journal of Digital Application \& Contemporary research, 2(1) (2013) 
21.Elbanby, G., El Madbouly, E., \& Abdalla, A.: Fuzzy principal component analysis for sensor fusion. In 11th IEEE International Conference in Information Science, Signal Processing and their Applications (ISSPA) pp. 442-447, IEEE, (2012)

22.Qader, k., \& Adda, M.: Network Faults Classification Using FCM. Paper presented at the 17th International Conference on "Distributed Computer and Communication Networks(DCCN-2013):" Control, Computation, Communication, Moscow (2013)

23.Wu, D., Yang, Q., Tian, F., \& Zhang, D. X.: Fault Diagnosis Based on K-Means and PNN. In 3rd International Conference on Intelligent Networks and Intelligent Systems (ICINIS), pp. 173-176). IEEE, (2010)

24. Specht, D. F.: Probabilistic neural networks. Neural networks, 3(1), 109-118 (1990)

25.Shahsavarani, S.: Probabilistic Neural Network, http://csewiki.unl.edu/wiki/index.php/Probabilistic Neural Network\#Architecture (2012) 